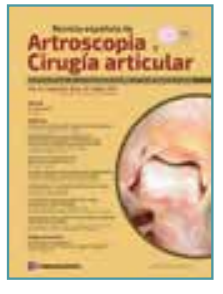

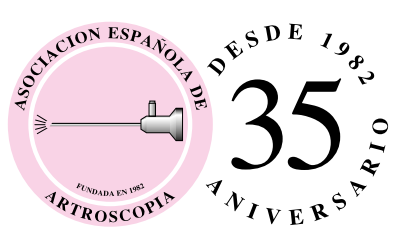

Vol. 24. Especial. Núm. 57. Mayo 2017

ISSN: 2386-3129 (impreso)

2443-9754 (online)

\title{
Original
}

\section{Cómo publicar un artículo científico paso a paso}

\author{
E. Sánchez Alepuz ${ }^{1,2}$, I. Miranda Gómez'1 A. Collado Sánchez¹, J.V. Díaz Martínez \\ 'Servicio de Cirugía Ortopédica y Traumatología. Hospital IMED Valencia. Burjassot, Valencia \\ 2 Servicio de Cirugía Ortopédica y Traumatología. Unión de Mutuas. Valencia
}

Correspondencia:

Dr. Eduardo Sánchez Alepuz

Correo electrónico: esancheza@telefonica.net
Recibido el 29 de enero de 2017

Aceptado el 6 de marzo de 2017

Disponible en Internet: mayo 2017

\section{RESUMEN}

Escribir un manuscrito para un artículo científico requiere un proceso de aprendizaje. Es importante conocer cómo escribir un manuscrito y el proceso de publicación para poder comunicar a la comunidad científica y, en concreto, en nuestro caso, a los especialistas en cirugía ortopédica y traumatología cualquier conocimiento que pueda hacer avanzar la especialidad. El objetivo del presente trabajo es exponer de forma sencilla cómo llegar a publicar un artículo científico, partiendo de la base de que se tiene algo que contar, para que lo que parece una montaña (escribir un manuscrito y publicarlo) se vea como un paso a paso y todo el que tenga algún conocimiento que pueda ser capaz de hacer avanzar a la ciencia lo pueda exponer a la comunidad científica.

Palabras clave: Manuscrito. Escribir. Publicar. Artículo científico. Factor de impacto.

\section{Introducción}

El objetivo de publicar un artículo científico debería ser el de poner en conocimiento de la comunidad científica algo que se piensa que puede ser útil para el desarrollo de la ciencia, la medicina o

\begin{abstract}
How to publish a scientific paper step by step

Writing a manuscript for a scientific article requires a learning process. It is important to know how to write a manuscript and the publishing process, in order to communicate to the scientific community, and specifically, in our case, to the specialists in orthopedic surgery and traumatology any knowledge that could make progress the specialty. The objective of the present work is to expose in a simple way how to publish a scientific article, on the basis that there is something to tell, so that what seems like a mountain (write a manuscript and publish it), could be seen as a step to step and anyone who has any knowledge that would be able to make science progress, could be able to expose it to the scientific community.
\end{abstract}

Key words: Manuscript. Writing. Publishing. Scientific article. Impact factor .

la especialidad ${ }^{(1)}$. Publicar no debería ser un objetivo en sí mismo, sino la consecuencia de un trabajo, pero escribir un manuscrito es un desafío, sobre todo para quien carece de experiencia ${ }^{(2)}$.

Los médicos asistenciales tienen/tenemos una sólida formación y experiencia clínica, tene-

https://doi.org/10.24129/j.reaca.24e57.fs1701005

FS $\odot 2017$ Fundación Española de Artroscopia. Publicado por Imaidea Interactiva en FONDOSCIENCE ${ }^{\circledR}$ (www.fondoscience.com). Este es un artículo Open Access bajo la licencia CC BY-NC-ND (www.creativecommons.org/licenses/by-nc-nd/4.0/). 
mos los conocimientos y tenemos muchas cosas que comunicar a la comunidad científica que podrían suponer un avance en nuestras especialidades, pero, en muchos casos, una escasa formación investigadora unida a la escasez de tiempo que se sufre por la exigente jornada asistencial hacen que esos conocimientos jamás se vean reflejados en un artículo en una revista, lo que supone un enlentecimiento del crecimiento de cada una de las especialidades y la pérdida de conocimientos. El objetivo del presente trabajo es exponer de forma sencilla cómo llegar a publicar un artículo científico, partiendo de la base de que se tiene algo que contar, para que lo que parece una montaña (escribir un manuscrito y publicarlo) se vea como un paso a paso y todo el que tenga algún conocimiento que pueda ser capaz de hacer avanzar a la ciencia lo pueda exponer a la comunidad científica.

\section{Algunas definiciones}

- Factor de impacto (FI): es una medida que pretende reflejar la importancia de una revista científica. Lo calcula año a año el Institute for Scientific Information (ISI) y los resultados son publicados en el Journal Citation Reports (JCR). El FI que tiene cada revista significa el número medio de veces que ha sido citado cada artículo de una revista concreta de los 2 años anteriores al año de publicación del JCR ${ }^{(3)}$. Por ejemplo, si una revista tiene un FI de 3,5 en el JCR de 2015, significa que los artículos publicados en esa revista entre 2013 y 2014 han sido citados una media de 3 veces y media en revistas que están incluidas en el propio JCR. Este valor numérico pretende establecer un valor del número de personas que tendrá acceso a un trabajo en función de la revista en que se publique, de forma que un mayor FI implicaría una difusión mayor de la revista ${ }^{(4)}$. Aunque este sea el índice bibliométrico más conocido, existen otros: SCImago Journal Rank (SJR), Source Normalized Impact per Paper (SNIP), etc. ${ }^{(4)}$.

- Journal Citation Reports (JCR): es el informe que emite cada año el ISI en el que otorga un FI a cada una de las revistas que es analizada. Este informe clasifica las revistas por especialidades, de forma que es posible comparar el FI de una revista con el de otras revistas similares en las que puede acceder a ser publicado un artículo determinado. Habitualmente, a la hora de evaluar a una revista se la clasifica, además de por el Fl, por el cuartil que ocupa dentro de las revistas de su especialidad. Por ejemplo, una revista con un FI de 2,5 en el JCR de 2015 clasificada en Pharmacology \& Pharmacy sería la revista 112 de 255 de las revistas de su grupo y, por tanto, se encontraría en el 2.o cuartil; en cambio, una revista con un FI de 2,5 en el JCR de 2015 clasificada en Orthopedics sería la revista 16 de 74 de las revistas de su grupo y, por tanto, se encontraría en el 1. ${ }^{\text {er }}$ cuartil, por lo que tendría una importancia relativa mayor en relación con la revistas de la especialidad.

- PubMed: es un buscador que da acceso libre a la base de datos MEDLINE de citas bibliográficas y resúmenes de artículos de investigación biomédica, que es ofrecido por la Biblioteca Nacional de Medicina de los Estados Unidos y recoge casi 5.000 revistas publicadas en los Estados Unidos y otros 70 países. El que una revista esté indexada en PubMed ofrece una visibilidad importante a la revista. Actualmente existen otras bases de datos de artículos de investigación: IME, EMBASE, Bibliomed, Scopus, IBECS, etc.

- Evaluación por pares (peer review): significa que la evaluación del manuscrito que enviamos a la revista la realiza uno o, en la mayoría de los casos, varios especialistas en la materia del manuscrito.

- Revistas open access: son revistas de libre acceso para los lectores desde Internet (a diferencia de las revistas tradicionales, a las que se accede por suscripción o a través de la suscripción de la institución -universidad, hospital, etc.- $)^{(6)}$. Existen 2 tipos, las que están sustentadas por alguna sociedad científica o grupo científico, que se hace cargo de los gastos, o aquellas en las que es el autor el que paga los gastos de publicación $^{(6,7)}$.

\section{Publicar un artículo científico paso a paso}

\section{Elección del tema y quiénes van a ser los autores del artículo}

Se debe elegir un tema que resulte de interés en la actualidad y en el que el investigador tenga algo que aportar: un detalle técnico, una serie importante de casos, resultados con una técnica novedosa, etc.; cualquier tema elegido puede ser 
bueno, siempre que el contenido del trabajo sea de calidad.

Para valorar quién o quiénes son los autores de un artículo, el International Committee of Medical Journal Editors (ICMJE; http://www.icmje. org $/)^{(8)}$ define los 4 criterios que debe cumplir un investigador para poder ser considerado coautor de una publicación:

1. Hacer una contribución sustancial al diseño del estudio o a la adquisición, análisis o interpretación de los datos del trabajo.

2. Escribir el manuscrito o hacer una revisión crítica del contenido del manuscrito.

3. Dar la aprobación a la versión final que se va a publicar.

4. Aceptar ser responsable de todos los aspectos del trabajo tras haberse asegurado de que cualquier cuestión relativa a la integridad de toda la obra ha sido estudiada y resuelta ${ }^{(1,8)}$.

\section{Búsqueda bibliográfica}

El investigador debe familiarizarse con las publicaciones relativas al tema que ha elegido y debe estar al tanto de qué se está publicando en los últimos meses-años ${ }^{(9)}$. Esto ayudará a establecer si el tema elegido es de interés y si lo que se quiere publicar aporta algo o ya está publicado. Además, la búsqueda bibliográfica sirve de punto de partida para plantear el objetivo, establecer la metodología y ayudar al investigador a elegir la revista a la que será enviado el manuscrito para su valoración. Una vez establecido el objetivo, deberá realizarse una segunda búsqueda bibliográfica más específica que continuará abierta y en revisión durante toda la redacción del manuscrito.

Para iniciar la búsqueda bibliográfica se debe elegir un buscador de citas bibliográficas, siendo PubMed el más utilizado, aunque existen otros. Una vez se va teniendo artículos de interés, la bibliografía citada en estos artículos también es una fuente importante que aporta al autor más referencias bibliográficas de interés. Se debe realizar una búsqueda bibliográfica crítica, ya que no todo lo que está publicado tiene por qué ser cierto $^{(9)}$. Se debe realizar una lectura crítica de los artículos científicos, analizar su metodología y sus resultados, se debe discriminar la calidad y relevancia de cada artículo, ya que tan malo es no incluir en la bibliografía artículos de relevancia para el tema que se va a tratar, como incluir artículos cuyo contenido sea cuestionable(9).

\section{Planteamiento del objetivo}

Debe realizarse de forma clara y concisa. Ayudará a los autores a un correcto desarrollo de la metodología y de la redacción del manuscrito. Habitualmente, el objetivo se escribe al final de la introducción. Muchos lectores buscan el objetivo del trabajo y esto determinará si siguen leyendo o no el texto.

\section{En qué revista lo quiero publicar}

Para elegir la revista donde se va a enviar un manuscrito, debemos tener en cuenta el tema del manuscrito y a qué público va dirigida la revista $^{(1,2)}$. El manuscrito tendrá mayor posibilidad de ser aceptado cuanto más se ajuste el tema al perfil de la revista donde es enviado ${ }^{(1,2)}$. A la hora de elegir una revista, debemos tener en cuenta qué público recibe la información (por ejemplo, la Revista Española de Artroscopia y Cirugía Articular llega directamente a todos los socios de la Asociación Española de Artroscopia -AEA- y además es accesible gratuitamente desde Internet), si la revista está indexada y dónde (PubMed, Scopus, etc.) y el Fl que tiene la revista ${ }^{(1,2)}$. Además debemos tener en cuenta el idioma en el que se publica la revista, ya que el idioma en que está escrito el artículo afecta a su probabilidad de ser citado, lo que indirectamente mide el impacto del artículo en la comunidad científica; en la actualidad, los artículos escritos en inglés tienen un mayor impacto en la comunidad científica ${ }^{(10)}$. Los autores deben valorar su pericia para escribir el artículo en su idioma nativo (el español en nuestro caso) o en inglés -existen empresas especializadas en la traducción de artículos científicos- y valorar en qué idioma escribir el manuscrito, porque aunque el inglés ofrece una mayor visibilidad para la comunidad científica en general ${ }^{(10)}$, los artículos escritos en español llegan con una mayor facilidad a los especialistas españoles y latinoamericanos; además de que en los últimos años, gracias a la calidad de su contenido, algunas revistas escritas en español están siendo indexadas en 
bases de datos internacionales, están ofreciendo simultáneamente la versión en inglés de los artículos y están teniendo cada vez mejores índices bibliométricos. En nuestra opinión, debemos encontrar un equilibrio entre publicar en revistas internacionales de habla inglesa y potenciar las revistas de nuestras sociedades científicas, ya que la única manera de que nuestras revistas mejoren sus indices bibliométricos (lo que se consigue si los artículos publicados son citados en otras publicaciones) es que se publiquen artículos de calidad en ellas, que puedan llamar la atención de otros autores, que potencialmente puedan citarlos en sus propios manuscritos.

\section{Diseño de la metodología}

Se debe ser cuidadoso y estricto en el diseño del trabajo que se va a realizar. Un error en el diseño puede provocar un error sistemático que invalide todos los resultados y las conclusiones del trabajo. Se debe establecer qué tipo de estudio se va a realizar: ensayo clínico, casos y controles, estudio de cohortes, etc.; cómo se va a seleccionar la muestra y cómo se va a distribuir a los sujetos en grupos (de manera sistemática, aleatoriamente, etc.); quién y cómo va a recoger los datos (puede ser conocedor o no del trabajo que se está realizando); quién y cómo va analizar los datos; se debe establecer qué parámetros estadísticos se van a medir y cómo se van a realizar las comparaciones entre grupos y entre qué grupos.

\section{Análisis de resultados}

Debe ser minucioso y concienzudo. La persona que analiza los datos puede saber o no la actuación que se ha hecho sobre cada grupo (si lo analiza de forma codificada, estará haciendo un análisis "ciego" de los datos). El análisis de los resultados debe responder a los objetivos marcados. Una vez analizados los resultados, los autores deben valorar si sus resultados tienen una coherencia interna (entre los propios resultados del trabajo) y externa (con otros resultados publicados). Si existe algún resultado inesperado, debe estudiarse por qué, valorar si es un error de la metodología o de la recogida de datos, y si todo es correcto, debe estudiarse una explicación.

\section{Redactar el manuscrito}

Debe ser un manuscrito estructurado y que se ajuste rigurosamente a las normas de la revista a la que va a ser remitido (en apartados, longitud, número de figuras, etc.). Con pequeñas modificaciones, los artículos científicos siempre tienen los mismos apartados:

- Introducción: debe ser breve y concisa. Tiene que incluir una breve descripción de lo que ya se conoce sobre el tema, los puntos que todavía no están resueltos o sobre los que existe una controversia y finalmente establecer un objetivo y una hipótesis para el trabajo que se está realizando(1).

- Métodos: debe ser detallado. Debe incluir toda la información necesaria para que otro investigador pueda reproducir el trabajo realizado $^{(2)}$.

- Resultados: se exponen los datos obtenidos en el trabajo, pero no debe incluirse su interpretación en esta sección $n^{(1,2)}$. Se deben incluir tablas y figuras, que deben estar referenciadas en el texto. No repetir datos en el texto que ya estén en las figuras o tablas y viceversa(1).

- Discusión: en esta sección deben interpretarse los datos obtenidos y deben compararse y relacionarse con lo ya conocido en la literatura publicada, y lo que es más importante, con lo que todavía no se conoce ${ }^{(1,2)}$. Se puede incluir un párrafo con las fortalezas y limitaciones del estudio ${ }^{(1)}$.

- Conclusión: algunas revistas lo incluyen al final de la discusión y otras como un apartado independiente. Exponer las conclusiones lógicas y explicar por qué estas conclusiones son importantes, cómo pueden influir en el pensamiento futuro en este y otros campos ${ }^{(2)}$. Todas las conclusiones deben desprenderse directamente de los resultados obtenidos en el trabajo.

- Bibliografía: debe estar referenciada toda la bibliografía citada en el texto. El formato debe ajustarse a las normas de la revista, tanto al formato de cada cita como al orden (algunas revistas utilizan el orden de aparición en el texto y otras lo hacen alfabéticamente). Hay revistas que limitan el número de referencias bibliográficas ${ }^{(1,2)}$.

Existen muchas maneras de escribir un manuscrito, pero en nuestra opinión, debe escribirse en primer lugar el objetivo, luego la sección de métodos y a continuación los resultados. Una vez los resultados estén escritos, debe comple- 
tarse la introducción y finalmente redactar la discusión y las conclusiones ${ }^{(2,9)}$. La bibliografía debe ir completándose conforme se va redactando el manuscrito.

\section{Resumen y título}

El resumen está marcado por las normas de la revista en cuanto a longitud (habitualmente entre 150 y 300 palabras) y en su estructura ${ }^{(1)}$. Ajustándose a las normas, el resumen debe incluir aquellos aspectos más relevantes del trabajo de una forma atractiva para el lector, ya que en la mayoría de las ocasiones, tras la lectura del resumen (y probablemente del objetivo), el lector decidirá si continúa leyendo el manuscrito completo ${ }^{(9)}$.

El título es la frase más importante de todo el trabajo y la que va a ser leída más veces, ya que muchos lectores leerán el título y solo unos pocos el resto del texto ${ }^{(9)}$. Es la primera impresión que se da al lector y determina si continuará leyendo el resumen ${ }^{(2,9)}$. Se deben utilizar palabras precisas, que indiquen el tema principal del artículo y que por sí solas ayuden a localizar el artículo en los buscadores cuando un investigador busque información sobre el tema ${ }^{(9)}$. Se ha demostrado que los artículos con títulos más cortos son más citados y que las revistas que publican artículos con títulos más cortos reciben más citaciones por artículo(11).

\section{Revisión del texto}

El primer paso de la revisión es la propia revisión, es decir, el autor que ha redactado el manuscrito debe revisarlo en profundidad una vez está completamente escrito. Se debe releer el manuscrito en su totalidad y desde el principio, como si fuese un manuscrito ajeno, revisar si los objetivos se plantean claramente y si se da respuesta a ellos a lo largo del manuscrito, evaluando la organización, el desarrollo y la distribución por párrafos ${ }^{(9)}$. Una vez el autor esté satisfecho con el contenido del manuscrito, debe hacer otra revisión, prestando especial atención a la ortografía, la gramática y los aspectos formales del manuscrito ${ }^{(9)}$.

En este momento, el manuscrito debe ser enviado a todos los coautores. El autor que ha redactado el manuscrito debe tener confianza, pero también objetividad y humildad para aceptar la crítica. Los coautores deben realizar una lectura crítica del manuscrito (como si fuesen los revisores de una revista), para señalar los errores y elaborar propuestas que mejoren el manuscrito final. Asimismo, puede resultar útil que revise el texto alguna persona que no haya participado en la elaboración del trabajo, que aportará información sobre si el manuscrito se entiende sin conocer los antecedentes y, si fuese necesario, algún especialista en alguno de los puntos (estadística, por ejemplo)(9). Cuanto mejor y más exhaustiva sea esta revisión interna, mayor calidad tendrá el manuscrito final.

\section{Enviar el artículo a la revista}

Actualmente, la mayoría de las revistas reciben los trabajos vía plataformas on-line y tienen su propio proceso de revisión. Se deben seguir las instrucciones de cada revista para la remisión de manuscritos ${ }^{(1,2)}$. Habitualmente, se debe remitir el manuscrito en 2 archivos: la "página del título", donde están los datos de los autores y sus filiaciones, y un segundo archivo, que es el "manuscrito ciego", que incluye el resto del manuscrito, sin ninguna referencia a los autores. Cada revista tiene sus propias normas respecto a la remisión de figuras y tablas. En ocasiones se requiere una carta de presentación, donde los autores deben exponer las fortalezas de su manuscrito y explicar qué aporta al conocimiento ya existente ${ }^{(1,2)}$.

\section{Respuesta del editor y los revisores}

Cada revista tiene unos tiempos para el proceso de revisión, pero se puede esperar una primera respuesta de 4 a 8 semanas después de la remisión del manuscrito (aunque en ocasiones es mucho más tiempo $)^{(1,2)}$. El autor recibirá una "carta de decisión". La decisión puede ser "aceptado" (lo que sucede excepcionalmente en una primera revisión), "revisión mayor" o "revisión menor", donde el editor y los revisores proponen una serie de cambios y mejoras a los autores, o "rechazado", donde el editor y los revisores explicarán los motivos (en este caso no hay que desanimarse, hay que revisar los motivos y preparar el manuscrito para enviarlo de nuevo a otra revista, si es posible 
mejorando los aspectos señalados por el editor o los revisores como causa de rechazo del artículo).

\section{Corrección del manuscrito}

En caso de recibir como respuesta que el manuscrito necesita "revisión mayor" o "revisión menor", se debe realizar esta revisión en cada uno de los puntos señalados por cada revisor. Debe elaborarse un documento de "respuesta a los revisores", que se adjuntará con el manuscrito modificado. El documento de "respuesta a los revisores" debe ser correcto y educado, se debe comenzar dando las gracias a los revisores y el editor por los comentarios, para a continuación contestar punto por punto cada uno de los comentarios de cada revisor. Se deben enumerar los comentarios y contestarlos uno a uno en el mismo orden en que aparecen en la carta de decisión. Se debe explicar qué modificación se ha hecho en el manuscrito en respuesta a cada comentario. Si en alguna de las propuestas de un revisor no se está de acuerdo, debe argumentarse educadamente, incluyendo bibliografía si es posible, evitando cualquier comentario despectivo o maleducado con el revisor. Por último, si los autores encuentran imposibilidad para realizar alguna de las modificaciones, deben explicar claramente los motivos ${ }^{(1,2,9)}$. Una vez completada la revisión, todos los coautores deben revisarla y dar el visto bueno.

\section{¿Qué sucede después de la aceptación del trabajo?}

\section{Prueba de impresión}

El autor de correspondencia recibirá una prueba de impresión del manuscrito en formato PDF, con las figuras y tablas ya montadas y el texto en el formato de la revista. Se debe revisar cuidadosamente, señalar a la revista si hay algún error y, si no lo hay, dar la aprobación al borrador.

\section{Publicación en "avance on-line" (Epub ahead of print)}

La mayoría de las revistas publican un "avance on-line", donde aparecen los artículos aceptados, que ya han pasado el proceso de producción y que por tanto están en su versión definitiva a falta de publicarse en uno de los números de la revista (por tanto, a falta de asignarles número de volumen y paginado). No obstante, en este momento el artículo ya puede ser citado, referenciado o ser incluido a todos los efectos en el currículum de los autores. Si es referenciado un artículo publicado como "avance on-line", la expresión "en prensa" o "epub ahead of print" sustituirá al volumen y paginado en la cita bibliográfica.

\section{Publicación del manuscrito definitivo}

El artículo finalmente será publicado en un número de la revista. A partir de este momento, se citará con el número de volumen y paginado correspondiente. El tiempo que tarda cada revista desde que un artículo es aceptado hasta que es publicado en su versión definitiva es muy variable.

\section{Visibilidad del artículo}

Dependerá de la revista donde esté publicado, de dónde esté indexada y de su FI. No obstante, y siempre cumpliendo las normas de copyright de cada revista, cada vez están adquiriendo mayor importancia las redes sociales científicas: Academia.edu, ResearchGate, Mendeley, Kudos, etc., que han aumentado la visibilidad de los trabajos científicos, aumentando la disponibilidad de los $\operatorname{artículos}^{(12)}$. En la mayoría de estas redes sociales, es el propio autor el que carga una copia de su artículo ${ }^{(12)}$. La más amplia y conocida de estas redes sociales es ResearchGate, con más de 9 millones de usuarios, donde los autores pueden cargar sus artículos desde las bases de datos bibliográficas o introducirlos manualmente, lo que permite una colaboración directa entre colegas y pares $^{(12)}$. Hay que tener precaución con las redes sociales científicas porque no controlan el contenido que cada autor sube a su perfil y, en los últimos años, han aparecido revistas fantasma con contenidos de muy baja calidad, que incluso falsifican datos de indices bibliométricos de la revista(13); sin embargo, si se usan con cuidado, estas plataformas pueden ayudar a los autores a dar visibilidad a sus trabajos y a poder contactar 


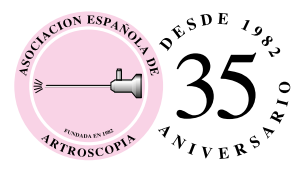

directamente con el autor de algún artículo que les resulte de interés, pudiendo contrastar opiniones o solicitar el texto completo en caso de no haberlo podido obtener por otros cauces.

\section{Conclusiones}

Existen muchas maneras de publicar un artículo científico, pero seguir este método paso a paso puede ayudar a investigadores inexpertos a publicar sus primeros artículos científicos y a hacerlo con un mayor rigor y un mejor resultado final.

\section{Bibliografía}

1. Vitse $C L$, Poland GA. Writing a scientific paper-A brief guide for new investigators. Vaccine. 2017. [Epub ahead of print].

2. O'Connor TR, Holmquist GP. Algorithm for writing a scientific manuscript. Biochem Mol Biol Educ. 2009; 37:344-8.

3. Nielsen MB, Seitz K. Impact Factors and Prediction of Popular Topics in a Journal. Ultraschall Med. 2016;37:343-5.

4. Mavrogenis AF, Megaloikonomos PD, Panagopoulos GN, Mauffrey C, Quaile A, Scarlat MM. Best one hundred papers of International Orthopaedics: a bibliometric analysis. Int Orthop. 2017. [Epub ahead of print].

5. Brown T. Journal quality metrics: options to consider other than impact factors. Am J Occup Ther. 2011; 65:346-50.

6. Poltronieri E, Bravo E, Camerini T, Ferri M, Rizzo R, Solimini R, Cognetti G. Where on earth to publish? A sample survey comparing traditional and open access publishing in the oncological field. J Exp Clin Cancer Res. 2013;32:4.

7. Björk BC, Solomon D. Open access versus subscription journals: a comparison of scientific impact. BMC Med. 2012;10:73.

8. International Committee of Medical Journal Editors. Defining the role of authors and contributors; 2015. Disponible en: http://www.icmje.org/recommendations/browse/ roles-and-responsibilities/defining-the-role-of-authors-andcontributors.html.

9. Davis, M, Davis KJ, Dunagan MM. Scientific Papers and Presentations. 3.a ed. Academic Press; 2012.

10. Di Bitetti MS, Ferreras JA. Publish (in English) or perish: the effect on citation rate of using languages other than English in scientific publications. Ambio. 2017;46:121-7.

11. Letchford A, Moat HS, Preis T. The advantage of short paper titles. R Soc Open Sci. 2015;2:150266.

12. Masic I, Begic E. Biomedical Scientific and Professional Social Networks in the Service of the Development of Modern Scientific Publishing. Acta Inform Med. 2016;24:409-12.

13. Memon AR. ResearchGate is no longer reliable: leniency towards ghost journals may decrease its impact on the scientific community. J Pak Med Assoc. 2016;66:1643-17. 